\title{
Circulating miRNAs as Potential Biomarkers of Age-Related Macular Degeneration
}

\author{
Chengda Ren ${ }^{a}$ Qingyu Liu ${ }^{\mathrm{a}}$ Qingquan Wei ${ }^{\mathrm{b}}$ Wenting Cai ${ }^{\mathrm{a}}$ Mengmei He \\ Yaru Duc Ding $\mathrm{Xu}^{\mathrm{a}}$ Yan $\mathrm{Wu}^{\mathrm{d}}$ Jing $\mathrm{Yu}^{\mathrm{a}}$ \\ aDepartment of Ophthalmology, Shanghai Tenth people's hospital, Tongji University School of \\ Medicine, Shanghai, 'Department of Ophthalmology, Shanghai Tenth people's hospital, Nanchang \\ University, Nanchang, 'Department of Ophthalmology, Shanghai Tenth People's hospital, Department \\ of First Clinical Medical College, Nanjing Medical University, Nanjing, dDepartment of Ophthalmology, \\ First Affiliated Hospital of Soochow University, Suzhou, People's Republic of China
}

\section{Key Words}

Age-related macular degeneration $\cdot$ microRNA $\cdot$ Microarray $\cdot$ Diagnosis $•$ Case-control

\begin{abstract}
Backgroud: Age-related macular degeneration (AMD) is one of the leading causes of irreversible blindness of the elder people. This research was intended to demonstrate the different expression of microRNAs (miRNA) in AMD patients and whether they can be used as biomarkers for AMD. Methods: MiRNAs expression was measured by microarray of 6 AMD cases and 6 gender matched controls. In a larger-sample case-control study with 126 AMD cases and 140 controls, whole blood samples were detected for the differences of miRNA expression. Results: A total of 216 differentially expressed miRNAs (111 increased and 105 decreased miRNAs) were detected from circulating miRNA microarray. Expanded case-control study results showed that the expression of miR-27a-3p, miR-29b-3p and miR-195-5p was increased significantly. Moreover, the level of miR-27a is higher in patients with wet AMD compared to patients with dry AMD. All 3 miRNAs showed a potential diagnostic value for AMD. Conclusion: Circulating miRNA levels were significantly varied in AMD patients. Three miRNAs, miR-27a-3p, miR-29b-3p and the miR-195-5p, might be potential diagnostic biomarkers for AMD.
\end{abstract}

\section{Introduction}

Age-related macular degeneration (AMD) is currently one of the leading causes of visual impairment and blindness throughout the world [1]. Genetic factors, aging, cigarette smoking, exposure to light and abnormal nutrition intake have been identified as AMD risk factors [2]. With the increasing number of elderly people in the population, the high prevalence of AMD was predicated to be a serious public problem for China in the future. According to the pathological type, the AMD cases were divided into 2 types: dry AMD and 
wet AMD [3]. Wet AMD was diagnosed when neurovascular vessels are detected. Anti-VEGF agent injection provided certain effects for the treatment of wet AMD; however, the long-term outcome should be determined in advanced studies [4]. Moreover, several clinical studies were conducted for the prevention of wet AMD arising from dry AMD, and no satisfactory results were detected. Thus, it was more important for the early diagnosis of AMD.

The diagnosis of AMD is currently determined by the clinical symptoms, fundus photography, optical coherence tomography (OCT) and fluorescein fundus angiography (FFA) [5]. Given that neither serious visual problems nor discomforts were present in dry AMD cases, it was hard to screen the early cases within the population. Furthermore, no effective prevention from the early to wet AMD has been determined after several clinical studies. Prognostic biomarkers for AMD were also lacking at the present time. The clinical situation of AMD highlighted the need for developing potential innovative biomarkers and therapeutic strategies. Identifying molecular factors associated with AMD incidence may lead to the identification of diagnostic biomarkers and the development of therapeutic targets that can improve the outcome of AMD cases.

Micro-RNAs (miRNAs) are a class of small non-coding RNAs consisting of 17-25 nucleotides [6]. In general, a miRNA would post-transcriptionally down-regulate the expression of its target genes through direct binding to the 3' untranslated region (3' UTR). Currently, a total of over 25,000 types of miRNAs have been identified in different species. Micro-RNAs produce various biologic effects, including cell proliferation, apoptosis, death and cell differentiation, to name a few. Recently, miRNAs have been suggested to participate in the development of the retina [7] and the incidence of retinal diseases [8]. Aberrant miRNA expression was also associated with the development of AMD [9]. Additionally, more studies were conducted to evaluate the use circulating miRNAs in the early detection of diversified diseases $[10,11]$. Considering these data, the detection of circulating miRNAs for AMD cases might be regarded as potential biomarkers and may lead to future advances in the treatment of AMD. The objective of this study was to determine whether circulating miRNAs could be a useful biomarker for the diagnosis of AMD cases using a miRNA microarray study. For further verification, a real-time quantitative reverse transcription polymerase chain reaction (RT-qPCR) method was conducted to explore whether miRNA expression could be measured reliably in blood samples from AMD cases. The diagnostic effects of certain miRNAs were studied for potential advanced clinical applications.

\section{Materials and Methods}

This study was approved by the local Human Research Ethics Committee of Tenth People's Hospital Affiliated to Tongji University before being conducted. All of the experiments described herein were in accordance with the guidelines and regulations issued by the Human Research Ethics Committee of Tenth People's Hospital Affiliated to Tongji University.

Patients and control individuals

In the microarray study, a total of 12 participants ( 6 experimental patients and 6 age- and gendermatched control subjects) were included. In the both AMD group and control group, 4 patients $(66.7 \%)$ were male, and 2 patients were female (33.3\%). The average age was $68.5 \pm 7.8$ years in the AMD group and was $65.7 \pm 6.9$ years in the control group. There was no significant difference in age between the two groups $(\mathrm{P}=0.525)$.

In the validation study, a total of 266 participants (126 AMD cases and 140 healthy subjects) were included. In the AMD group, there were 67 male and 59 female patients. However, in the control group, there were 74 males and 66 females. No statistically significant difference in gender between both groups was detected $(\mathrm{P}=0.528)$. The average age was $58.4 \pm 11.2$ years in the AMD group and was $54.9 \pm 10.7$ years in the control group. No statistically significant difference in the age distribution was detected $(P=0.010)$.

Blood samples were obtained from all of the participants in the study. The samples were obtained in the outpatient department of the Tenth People's Hospital Affiliated to Tongji University between January 


\section{Cellular Physiology Cell Physiol Biochem 2017;41:1413-1423

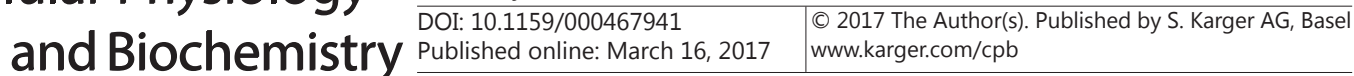 \\ Ren et al.: Circulating miRNAs and AMD}

2014 and November 2016. The experiments were undertaken with the understanding and written consent of each subject. The study methodologies conformed to the standards set by the Declaration of Helsinki and the study methodologies were approved by the local ethics committee. The diagnosis of AMD was based on the clinical symptoms, fundus photography, OCT and FFA examinations. The controls were healthy subjects that were age- and gender-matched in this study. The exclusion criteria were as follows: (1) high myopia, recent diagnosed conjunctivitis, keratitis and uveitis; (2) patients with macular hole, macular edema, retinal vascular occlusion or central serous choroid retinopathy; (3) recent diagnosed infection, hypertension, diabetes, cancer, Alzheimer's disease, stroke, autoimmune diseases and other organic diseases; or (4) any patients who failed to complete of the relevant inspection and research.

\section{RNA extraction}

In both the microarray study and validation study, blood samples were transferred to $-80{ }^{\circ} \mathrm{C}$ within half an hour of sample collection. For the microarray study, total RNA from 12 samples was extracted from whole blood using TRIzol reagent (Invitrogen, Carlsbad, CA, USA) according to the instructions provided by the manufacturer. For the validation study, RNA extraction was conducted on ice using a miRNAeasy kit (Qiagen, Valencia, CA, USA) according to the manufacturer's protocol. After RNA extraction, the purity of the isolated RNA was determined using the OD260/280-reading tool on a NanodropND-2000 spectrophotometer (Thermo Scientific, Worcester, MA, USA).

\section{Microarray profiling}

A total of 12 RNA samples were labeled using the miRCURY Hy3/Hy5 Labeling Kit after quality control. The processed RNA samples were hybridized with miRCURY (v.18.0) for microarray hybridization. After the microarray was cleaned, all of the chips were analyzed with the analysis system (genepix Pro 6.0 software, axon). All of the data from the 12 microarrays were normalized with non-control probes whose values were equal to or larger than 30 . The median of these non-control probes was used as a normalization factor. The formula used was as follows: standard value = each miRNA correction value $/$ median.

\section{RT-PCR analyses}

Taqman miRNA assays were adopted to detect the expression circulating miRNA in the whole blood samples. All products, including reagents, primers, and probes were obtained from Applied Biosystems (Foster City, CA, USA). A total of 120 ng of total RNA from all the samples were added in 15 - $\mu$ L reactions containing reverse transcription (RT) mixture and primers. The mix was incubated at $94{ }^{\circ} \mathrm{C}$ for $2 \mathrm{~min}$, then $94{ }^{\circ} \mathrm{C}$ for $20 \mathrm{sec}$ and $60{ }^{\circ} \mathrm{C}$ for $34 \mathrm{sec}$. All the experiments were conducted using the Mx3005P qPCR System (Agilent, Santa Clara, CA, USA). The relative expression of the mature miRNA was calculated using the comparative cycle threshold $(2-\Delta \Delta \mathrm{CT})$ method. The 18S RNA was used as an endogenous control for advanced normalization of data.

We used Linear Models for Microarray Data (LIMMA), a package for the analysis of gene expression data arising from microarray or RNA-Seq technologies [12]. P values of less than 0.01 were considered significant in the detection of differentially expressed micro-RNA detection. The heatmap of differentially expressed micro-RNA was generated with R bioconductor.

Bioinformatics analysis

In the present study, we used miRBase (http://www.ebi.ac.uk/enright-srv/microcosm/htdocs/ targets/v5/Microcosm), Miranda (Http://www.microrna.org/microrna/home.do hg19, Miranda) and targetscan (http://www.targetscan.org/vert_60/) as target gene forecast strategies. The functional enrichment analysis was conducted by independent studies. DAVID (the Database for Annotation, Visualization and Integrated Discovery), a high throughput and integrated data-mining environment, was the most popular tool to systematically extract biological meaning from large gene or protein lists. The GO terms were analyzed and then plotted using Gene Ontology Annotation Plot. Moreover, target genes were also annotated using Kyoto Encyclopedia of Genes and Genomes.

\section{Data analysis}

The differential expression levels of circulating miRNAs between AMD patients and healthy control subjects were analyzed using a two-tailed t-test. The data in the study are presented as the mean value plus or minus standard deviation (mean $\pm \mathrm{SD}$ ). Validation of the miRNA diagnostic effect of each miRNA and from the three selected differently expressed miRNAs was conducted by receiver operating characteristic 


\section{Cellular Physiology Cell Physiol Biochem 2017;41:1413-1423

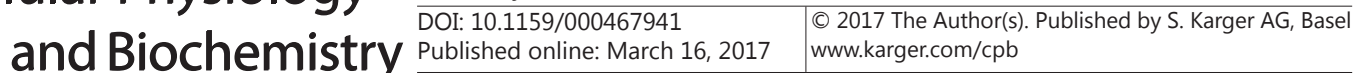 Ren et al.: Circulating miRNAs and AMD}

(ROC) curves analysis. Optimal cutoff by highest sensibility plus specificity and efficiency values were determined. ROC analysis for miRNAcombined, geometric means of expression levels was calculated using three miRNAs selected with the highest efficiency values. $\mathrm{P}<0.05$ were considered statistically significant. The data analyses were performed using SPSS software version 16.0 (SPSS Inc., Chicago, IL, USA).

\section{Results}

\section{Differently expressed miRNAs}

The results of cluster analysis of the microarray show that compared with the controls, 216 circulating miRNAs were differently expressed (increased 111 miRNAs increased and 105 miRNAs reduced). Based on the large amount of differently expressed miRNAs, the P value was set at less than 0.05 , and the fold change was set to greater than two fold for further analysis. A total of 35 miRNAs were differently expressed. Among these, 15 miRNAs were up-regulated, and 20 miRNAs were down-regulated. The detailed data are presented in Fig. 1 and Table 1.

\section{Bioinformatics analysis}

GO analysis showed that the target genes of different miRNAs were processed. The genes which related to cellular process (681), single-organism process (635) and metabolic processes (601) changed significantly. It indicated that the aberrantly expressed miRNAs mainly involved in regulating the biological process mentioned above (Fig. 2A). To make sure which pathways play a critical role, we proceeded pathway analysis based on target genes of miRNAs. (Database: http://www.kegg.jp/) Results showed that cell cycle pathway had the highest correlation with AMD and the correlation extent is dramatically higher than other related pathways. It indicated that dysregulation of cell cycle might participate AMD progression.

\section{Case-control validation study}

In the present study, a total of 126 AMD patients (72 dry AMD and 54 wet AMD cases) and 140 control subjects were included. Among all of the differently expressed miRNAs from microarray assay, 10 miRNAs were selected under an integrated
Table 1. MiRNAs differently expressed from microarray analyses

\begin{tabular}{llll}
\hline \multicolumn{2}{c}{ Up-regulated } & \multicolumn{2}{c}{ Down-regulated } \\
miRNA name & Fold & \multicolumn{1}{c}{ miRNA name } & Fold \\
\hline hsa-miR-644b-5p & 4.92 & hsa-miR-513c-3p & 0.13 \\
hsa-miR-559 & 3.71 & hsv2-miR-H9-3p & 0.29 \\
hsa-miR-152 & 3.66 & hsa-miR-30c-1-3p & 0.31 \\
hsa-miR-195-5p & 3.41 & hsa-miR-4723-3p & 0.33 \\
hsa-miR-27a-3p & 2.63 & hsa-miR-3614-3p & 0.34 \\
hsa-miR-126-5p & 2.51 & hsa-miR-3195 & 0.36 \\
hsa-miR-4638-5p & 2.43 & hsa-miR-4305 & 0.38 \\
hsa-miR-28-5p & 2.34 & hsa-miR-328 & 0.38 \\
hsa-let-7c & 2.33 & hsa-miR-4324 & 0.38 \\
hsa-miR-5189 & 2.29 & hsa-miR-4658 & 0.38 \\
hsa-miR-1273f & 2.15 & hsa-miR-584-5p & 0.41 \\
hsa-miR-513b & 2.08 & hsa-miR-2114-5p & 0.41 \\
hsa-miR-25-3p & 2.07 & hsa-miR-186-3p & 0.44 \\
hsa-miR-149-3p & 2.01 & hsa-miR-5571-5p & 0.45 \\
hsa-miR-29b-3p & 2.00 & hsa-miR-18b-3p & 0.45 \\
& & hsa-miR-4667-3p & 0.45 \\
& & hsa-miR-4653-3p & 0.46 \\
& & hsv1-miR-H14-3p & 0.46 \\
& & hsa-miR-4436b-5p & 0.46 \\
& & hsa-miR-1470 & 0.47 \\
\hline
\end{tabular}

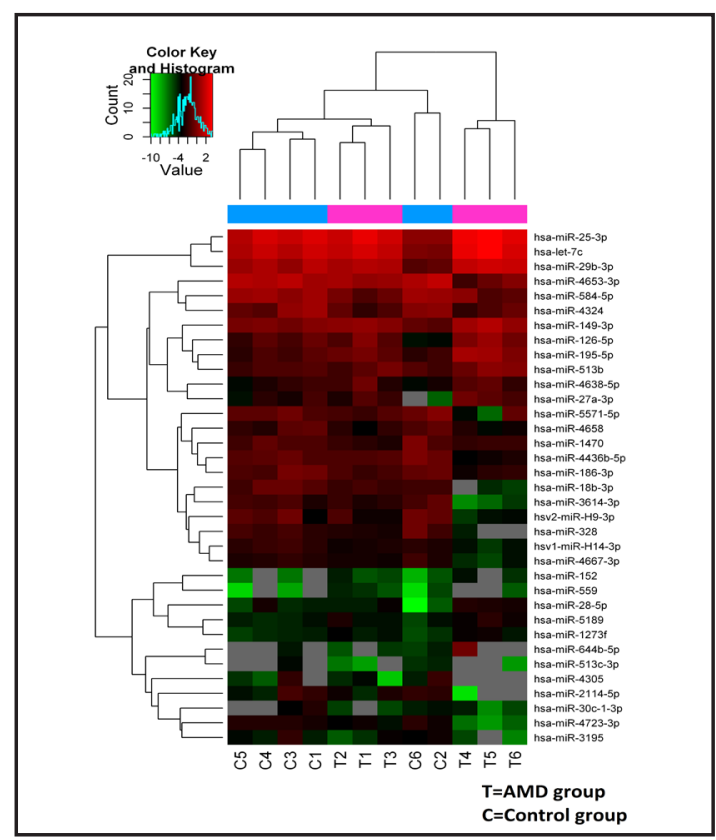

Fig. 1. The heat map of miRNA expression in the AMD cases compared with the controls. The heat map shows the expression of 35 candidate miRNA marker which are demonstrated significant differences between 6 AMD patients and 6 healthy controls. Each column stands for an individual sample, column of C1-C6 are controls and column of T1-T6 are AMD patients. Each row corresponds to different plasma miRNA. MiRNAs are represented in red when upregulate while in green when downregulate. 


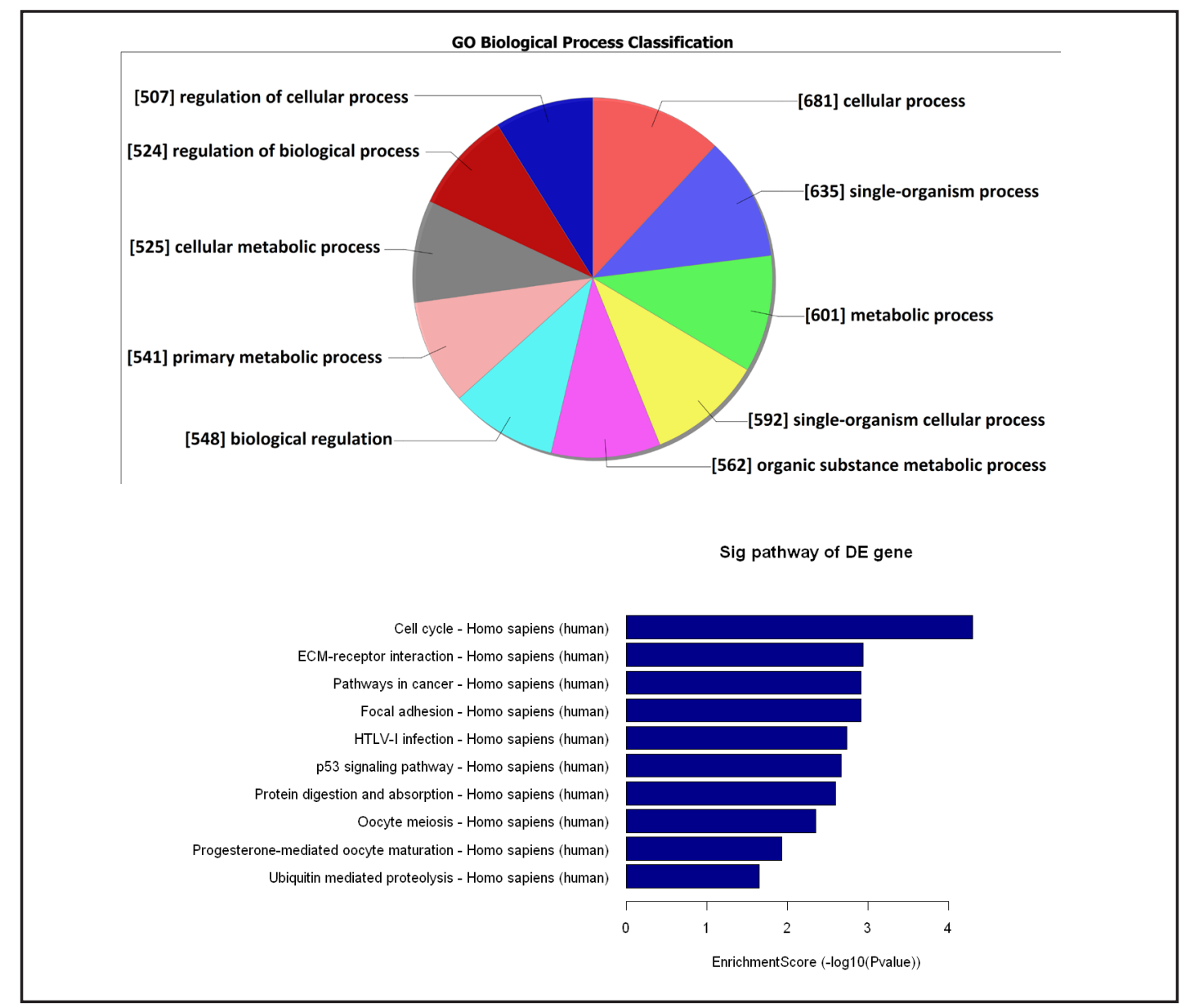

Fig. 2. The most related biological progresses and pathways with AMD pathogenesis. Fig 2A shows the GO biological progresses classification of the target genes of the differently expressed miRNAs. The area of each part represent the amount of target genes act in different biological processes. The 3 most biological processes which are related with different expressed miRNAs are cellular process (681 genes), single-organism process (635 genes) and metabolic processes (601 genes). Fig 2B demonstrates that cell cycle pathway is the most related pathway to AMD which shows a significant higher correlation than other pathways.

consideration of their target genes and expression differences (up-regulated: miR-152, miR27a-3p, miR-28-5p, miR-195-5p, miR-559, miR-25-3p, miR-29b-3p and hsa-let-7c; downregulated: miR-328 and miR-584-5p). Compared with the control group, it was found that the expression of miR-27a-3p, miR-29b-3p and miR-195-5p were significantly increased, and the increases ratios were $2.68 \pm 1.00,3.53 \pm 1.78$ and $4.23 \pm 3.08$ times, respectively. However, there were no significantly different changes found in other miRNAs between the two groups. All of the detailed data are presented in Fig. 3A. Additionally, we analyzed the miRNA-miRNA network of the miRNAs we selected and their target genes (Fig. 3B).

\section{Micro-RNA expression in different AMD stages}

To further verify the relative expression of the three differently expressed miRNAs (miR-27a-3p, miR-195-5p and miR-29b-3p), we found that all 3 miRNAs were up-regulated in both dry AMD and wet AMD cases. In addition, we found that miR-27a showed a higher expression in the wet AMD cases compared with the dry AMD cases. However, no significant differences of miR-29b-3p and miR-195-5p expression were detected between the different AMD stages (Fig. 4). 


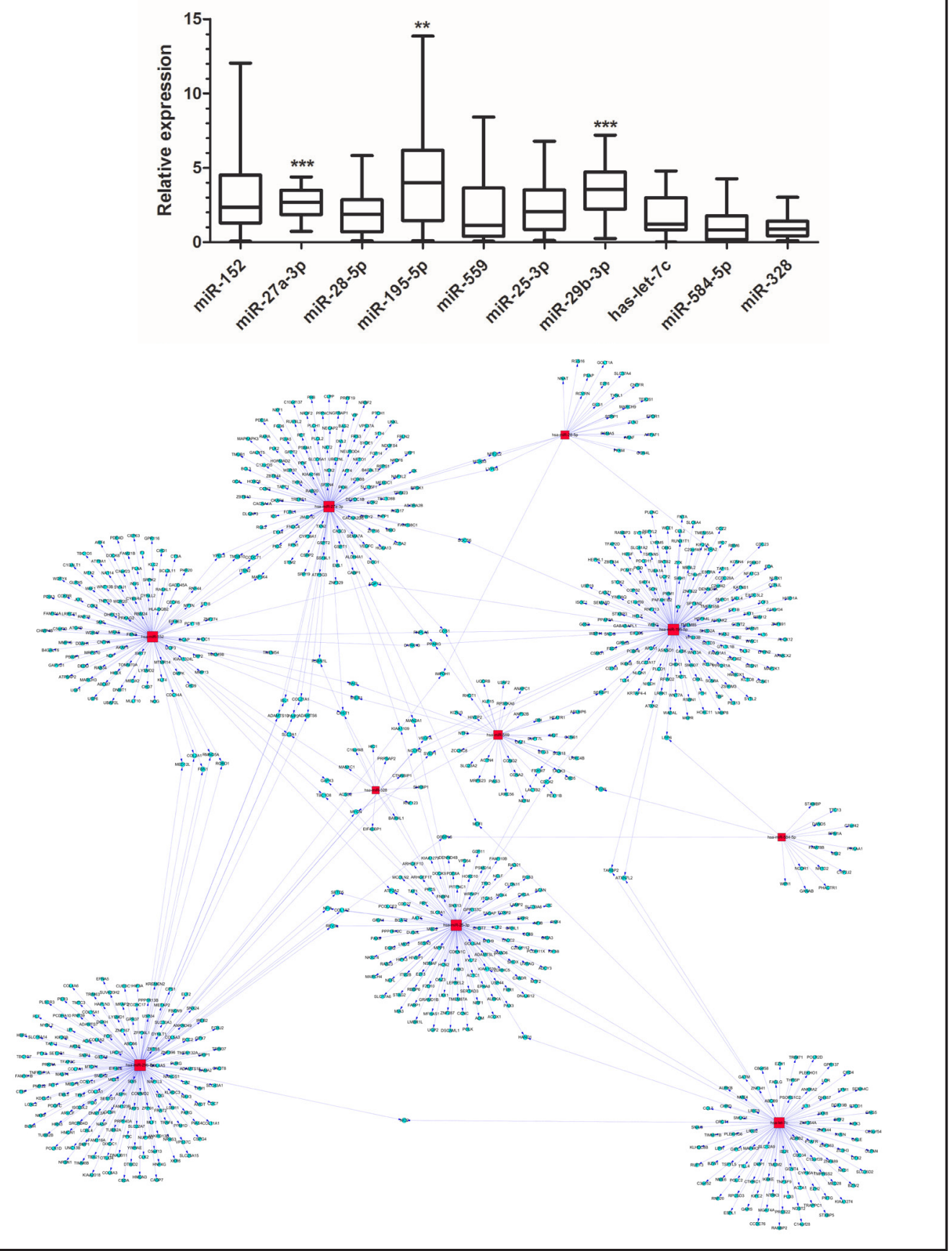

Fig. 3. (A) The relative expression of 10 miRNAs (miR-152, miR-27a-3p, miR-28-5p, miR-195-5p, miR-559, miR-328, miR-25-3p, miR-29b-3p, has-let-7c, miR-584-5p) in a larger-sample case-control study with 126 AMD cases and 140 controls. In which miR-27a-3p, miR-195-5p and miR-29b-3p are upregulated with a statistical significance $\left({ }^{* *} \mathrm{p}<0.01,{ }^{* * *} \mathrm{p}<0.001\right)$. (B) Network of miRNAs and their predicted target genes.

The diagnostic effect of differently expressed miRNAs

We further analyzed the diagnostic effects of three differentially expressed miRNAs by ROC analyses among 126 AMD cases and 140 controls. The miR-27a-3p, miR-29b-3p and miR-195-5p expression levels were detected by RT-PCR methods for each participant. All the data were imported into SPSS software and the ROC curve was used for the diagnostic KARGER 
Fig. 4. Relative expression of miR-27a-3p, miR-195-5p and miR-29b-3p in different stages of AMD cases (wet AMD, $n=54$, dry AMD, n=72). Only miR-27a-3p demonstrates a higher expression in wet AMD group than dry AMD group $(\mathrm{p}<0.001)$.
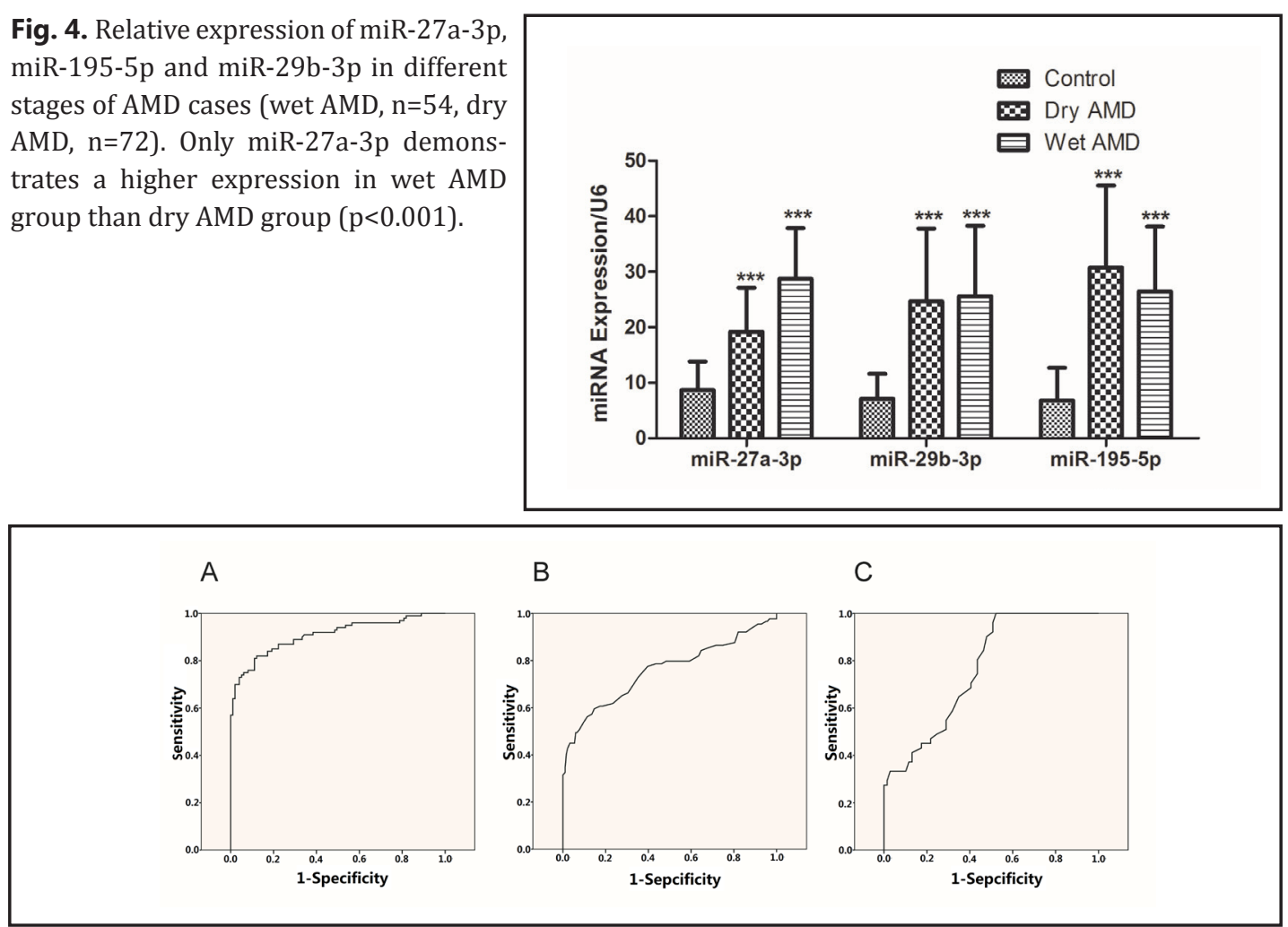

Fig. 5. The ROC curve of miR-27a-3p, miR-195-5p and miR-29b-3p for the diagnosis of AMD. (A, B, C) ROC analysis of the logistic regression model based on plasma miR-27a-3p, miR-195-5p and miR-29b-3p for AMD patients $(n=126)$ from healthy controls $(n=140)$. AUC of each miRNA is $0.925,0.757$, and 0.766 separately.

effect of differently expressed miRNAs independently. According to the ROC curve analyses, we found that all three miRNAs could be invoked as diagnostic markers of AMD. As shown in Fig. 5, the areas under the curve (AUC) of miR-27a-3p (Fig. 5A), miR-29b-3p (Fig. 5B) and miR-195-5p (Fig. 5C) were $0.925(95 \% \mathrm{CI}=0.892-0.963)$ and $0.757(95 \% \mathrm{CI}=0.679$ $0.832)$ and $0.766(95 \% \mathrm{CI}=0.672-0.813)$, respectively. The results suggest that circulating miR-27a-3p, miR-29b-3p and miR-195-5p demonstrated the potential for use as diagnostic biomarkers of AMD with 0.825 sensitivity and 0.728 specificity when miR-27a-3p was $\geq 12.0$, 0.688 sensitivity and 0.718 specificity when miR-29b-3p was $\geq 10.6$, and 0.726 sensitivity and 0.596 specificity when miR-195-5p was $\geq 10.2$.

\section{Discussion}

AMD is the most influential cause of irreversible visual acuity loss in people over 50 years of age in western countries. Only mild symptoms are presented in dry AMD cases; however, the visual acuity will be markedly impaired when it progresses to the wet AMD stage. Moreover, there are no ideal treatments for AMD, and thus effective markers for early diagnosis and prognosis of AMD are urgently needed. At present, sensitive biomarkers for early diagnosis and effective prognosis are lacking. In our study, we identified three differently expressed miRNAs in AMD patients who may be used as potential biomarkers for AMD. Our research remedied the lack of high-throughput analysis of circulating miRNAs in cases of AMD. We also demonstrated a profile of variation of miRNA levels in AMD patients that played a role in guiding studies of AMD biomarkers.

miRNAs have been found to regulate variant of cell functions and participate a number of pathways. The disorder of these functions in retinal cells may contribute to AMD 
progression. Recent studies have indicated that following miRNAs are associated with the development of AMD: the miR-17 cluster, miR-21, miR-27, miR-132, miR-204, miR-210, miR296, miR-378, miR-519c and the miR-15/107 group [13, 14]. Disregulation of these miRNAs in ocular may result in different secretion of miRNAs in serum. Therefore, it is valuable to investigate miRNAs expression change in serum and establish noninvasive biomarkers. There are several studies have demonstrated a number of AMD biomarkers, however an accepted one is still lacking.

Menard et al. found that miR-146a was up-regulated in vitreous humour of wet AMD patients [15]. The expression profile analysis of miRNAs in vitreous humour is important in further understanding AMD pathogenesis. However, the results still need replication in larger sample size and in other racial. Another study showed that miR-661 and miR3121 were expressed at significantly higher levels in dry AMD; miR-4258, miR-889 and Let-7 in wet AMD [16]. Significantly, the found that there is a positive correlation between the expression of VEGF and VEGFR2 on both the mRNA and the protein levels, and the expression of Let-7 in wet AMD patients. Let-7 family members are known to be proangiogenic miRNAs. Their findings strongly suggest that the up-regulation of Let-7 in serum may reflect the neovascularization in AMD patients. Grassmann et al. found that miR-3013p, miR-361-5p and miR-424-5p were down-regulated in NV AMD patients compared with controls. To evaluate subtype-specificity, 59 more AMD cases with geographic atrophy (GA) were analyzed for the miR-301-3p, miR-361-5p and miR-424-5p. No significant differences were found between GA AMD and controls. These results indicate that the three miRNAs mentioned above may participate in wet AMD pathogenesis, whereas the exact mechanism remains unknown [17]. Ertekin et al. analyzed some differently expressed miRNAs in AMD patients [18]. There were some interesting findings that were consistent with ours. They demonstrated that miR-27b-3p, miR-29a-3p and miR-let-7c were expressed in only the patient group, and we found that miR-27a-3p, miR-29b-3p and miR-let-7c were significantly up-regulated in the patient group of our microarray study. Our results indicated that the miR-27 and miR-29 family and miR-let-7c might be potential targets for further studies of AMD biomarkers. However, the majority of the miRNAs that Ertekin et al. found to be upregulated or down-regulated in AMD patients were different from the results of our study. These differences may be due to insufficient sample capacities and the use of different races in the two studies.

Results of microarray profiles in this study showed that circulating miRNAs were differently expressed in the AMD group compared with the controls. When the miRNAs with fold changes greater than 2 were considered, a total of 35 miRNAs (15 up-regulated and 20 down-regulated) were detected in this study. However, the result is not stable enough. For example, miR-644-5p, which has the highest fold change but the changing fold was pulled up by one patient. Considering that the variant of miRNA expression, we choose ten miRNAs which expression levels are significant and stable. However, it does not mean other miRNAs are insignificance. More studies need to be conducted to verify the results. Advanced bioinformatics analysis showed that the differently expressed circulating miRNAs were mainly involved in cellular process, biological regulation and metabolic processes, which indicated that cellular process dysfunction is the main pathogenesis of AMD. Moreover, functional enrichment analysis of target genes indicated that cell cycle was the most related pathway of AMD pathogenesis. The results of pathway analysis confirmed that cellular process is dysregulated in AMD patients. $A \beta$, a known constituent of drusen in AMD patients, was found to arrest cell cycle and cause cell senescence which is concordant with our KEGG pathway results [19]. However, there has not been a thorough study of AMD progression and cell cycle dysfunction. Our results provided a new direction to demonstrate the development of AMD.

Although the microarray profiles have the advantages of fast, stable and high throughput results, they usually contain a small sample size. Consequently, we conducted an advanced case-control study with relatively larger samples. The results from miRNA microarray profiling were validated when using the detection of significant results from a larger sample 
size. A total of 10 detected miRNAs in the microarray results (miR-152, miR-27a-3p, miR-285p, miR-195-5p, miR-559, miR-25-3p, miR-29b-3p, has-let-7c, miR-328 and miR-584-5p) were selected for further study. Consistent with the results of the microarray, the expression of miRNA expression from the case-control study exhibited similar trends. Within the ten selected miRNAs, statistically significant differences were detected for three miRNAs. In general, miR-27a-3p, miR-29b-3p and miR-195-5p were increased by $2.68,3.53$ and 4.23 times, respectively, in AMD cases compared to controls. Only miR-27a-3p demonstrated different expression levels between the dry and wet AMD groups and thus suggests a further potential of miR-27a-3p as a marker for the development of AMD. ROC curve analysis of the three miRNAs also showed that the 3 miRNAs could be used as biomarkers for AMD. In this study, the circulating miR-27a-3p demonstrated the highest sensitivity (0.825), and the highest specificity (0.728).

The change in miRNA expression always indicates a class of abnormal function such as pathological angiogenesis, oxidative stress response, immune response and inflammation. Through the comparison of retinal tissues from the AMD and control groups, it was found that miR-23a expression was markedly decreased in the retina of AMD patients. An unconventional in-vitro study showed that miR-23a could reduce the oxidative damage induced by hydrogen peroxide and thus exhibited protective effects [20]. Another study of CNV in a laser induced CNV model by Zhou et al. showed that microRNA-23 27 24 clusters play an important role in angiogenesis [21]. They demonstrated that miR-27 and miR-23 are required for pathological angiogenesis in a laser-induced choroidal neovascularization mouse model. Additionally, inhibiting the expression of miR-27 and miR-23 could decrease the angiogenesis progression. The results of these studies show that miRNAs could participate in AMD pathogenesis and demonstrate definite clinical significance.

Information concerning the effects of the three mentioned miRNAs (miR-27a-3p, miR29b-3p and miR-195-5p) in AMD development is still lacking. MiR-27a, which is located on chromosome 19p13.13, demonstrates a capital effect in tumor formation, cell proliferation, apoptosis and differentiation [22]. A previous study reported that miR-27 could influence brain development through inhibiting apoptotic protease activating factor 1 (APAF-1) and thus regulated the sensitivity of the brain cells to apoptosis [23]. The up-regulation of miR-27a-3p that we detected is in accordance with Zhou's results. Upon further analysis, we detected a higher expression of miR-27a-3p in wet AMD than dry AMD. The cause of such a difference may be attributed to the CNV accompanying miR-27a-3p up-regulation in wet AMD. Thus, up-regulation of circulating miR-27a-3p has a strong possibility of indicating CNV formation. MiR-29-3p and miR-195-5p are located on chromosomes 18 and 17, respectively. Both miRNAs demonstrate important effects on cell differentiation and tumor formation [24]. Furthermore, they were reported to be important factors in the diagnosis and prognosis of several diseases [25-27]. It is interesting to see that VEGF is a congenerous target gene of both miR-29b-3p and miR-195-5p. A possible mechanism of the increased expression of miR-29b-3p and miR-195-5p in the circling samples could be a reaction of the aging activation. In a study based on a rat model of diabetic nephropathy, miR-29b was reported to be a new therapeutic molecule and reversed the progression of diabetic nephropathy. In diabetic nephropathy, the mechanism of action of miR-29b occurs through its anti-inflammatory effect and inhibition of fibrosis [28]. Considering that the data in this study were based on whole blood samples of AMD patients, the changes in circulating miRNA content might be not completely consistent with local changes. More analysis should be performed before conducting advanced in-vitro studies. Presently, fresh retinal samples of AMD patients, especially in dry AMD cases, are hard to obtain; therefore, we must rely on a more prominent role for circulating miRNA detection.

Numerous limitations existed in this study. First of all, the sample size is not large enough. Secondly, the long-term follow-up data were lacking in this study. It was difficult to determine the prognostic effect of circulating miRNAs in AMD development. Thirdly, even though several specific miRNAs with potential effects were detected in this study, the detailed mechanisms should be studied in further analyses. In the future, a larger sample 


\section{Cellular Physiology Cell Physiol Biochem 2017;41:1413-1423

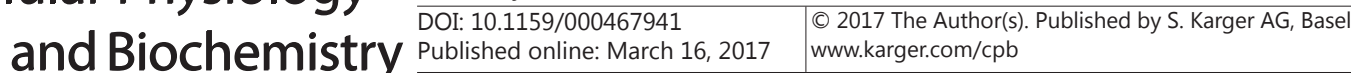 \\ Ren et al.: Circulating miRNAs and AMD}

size study should be conducted, and the comparison of the expression in tissue and blood samples should be examined. The exact role of miRNAs on the pathogenesis of AMD should also be examined in future studies.

\section{Acknowledgements}

This work was supported by the National Natural Science Foundation of China (No 81470648) and Scientific Research Project of Shanghai Sanitary Bureau (No 20124100).

\section{Disclosure Statement}

The authors declare no competing financial interests.

\section{References}

1 Schaal KB, Rosenfeld PJ, Gregori G, Yehoshua Z, Feuer WJ: Anatomic Clinical Trial Endpoints for Nonexudative Age-Related Macular Degeneration. Ophthalmology 2016;123:1060-1079.

-2 Lancon A, Frazzi R, Latruffe N: Anti-Oxidant, Anti-Inflammatory and Anti-Angiogenic Properties of Resveratrol in Ocular Diseases. Molecules 2016;21:304.

-3 Luttrull JK, Margolis BW: Functionally Guided Retinal Protective Therapy for Dry Age-Related Macular and Inherited Retinal Degenerations: A Pilot Study. Invest Ophthalmol Vis Sci 2016;57:265-275.

-4 Eandi CM, Alovisi C, De Sanctis U, Grignolo FM: Treatment for neovascular age related macular degeneration: The state of the art. Eur J Pharmacol 2016;787:78-83.

-5 van Lookeren Campagne M, Strauss EC, Yaspan BL: Age-related macular degeneration: Complement in action. Immunobiology 2016;221:733-739.

6 Zuo K, Zhi K, Zhang X, Lu C, Wang S, Li M, He B: A dysregulated microRNA-26a/EphA2 axis impairs endothelial progenitor cell function via the p38 MAPK/VEGF pathway. Cell Pysiol Biochem 2015;35:477488.

7 Karali M, Persico M, Mutarelli M, Carissimo A, Pizzo M, Singh Marwah V, Ambrosio C, Pinelli M, Carrella D, Ferrari S, Ponzin D, Nigro V, di Bernardo D, Banfi S: High-resolution analysis of the human retina miRNome reveals isomiR variations and novel microRNAs. Nucleic Acids Res 2016;44:1525-1540.

-8 Wu J, Wang R, Ye Z, Sun X, Chen Z, Xia F, Sun Q, Liu L: Protective effects of methane-rich saline on diabetic retinopathy via anti-inflammation in a streptozotocin-induced diabetic rat model. Biochem Biophys Res Commun 2015;466:155-161.

-9 Bhattacharjee S, Zhao Y, Dua P, Rogaev EI, Lukiw WJ: microRNA-34a-Mediated Down-Regulation of the Microglial-Enriched Triggering Receptor and Phagocytosis-Sensor TREM2 in Age-Related Macular Degeneration. PLoS One 2016;11:e0150211.

-10 Sun Y, Liu Y, Cogdell D, Calin GA, Sun B, Kopetz S, Hamilton SR, Zhang W: Examining plasma microRNA markers for colorectal cancer at different stages. Oncotarget 2016;7:11434-11449.

$>11$ Chang PY, Chen CC, Chang YS, Tsai WS, You JF, Lin GP, Chen TW, Chen JS, Chan EC: MicroRNA-223 and microRNA-92a in stool and plasma samples act as complementary biomarkers to increase colorectal cancer detection. Oncotarget 2016;7:10663-10675.

12 Smyth GK: Linear models and empirical bayes methods for assessing differential expression in microarray experiments. Stat Appl Genet Mol Biol 2004;3:Article3.

13 Machalinska A, Kawa MP, Marlicz W, Machalinski B: Complement system activation and endothelial dysfunction in patients with age-related macular degeneration (AMD): possible relationship between AMD and atherosclerosis. Acta Ophthalmol 2012;90:695-703. 


\section{Cellular Physiology Cell Physiol Biochem 2017;41:1413-1423

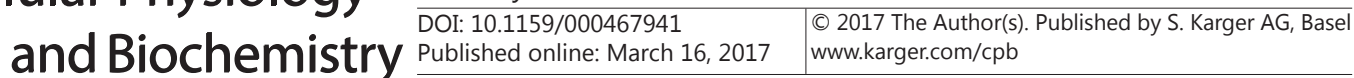

Ren et al.: Circulating miRNAs and AMD

14 Wang S, Koster KM, He Y, Zhou Q: miRNAs as potential therapeutic targets for age-related macular degeneration. Future Med Chem 2012;4:277-287.

15 Menard C, Rezende FA, Miloudi K, Wilson A, Tetreault N, Hardy P, SanGiovanni JP, De Guire V, Sapieha P: MicroRNA signatures in vitreous humour and plasma of patients with exudative AMD. Oncotarget 2016;7:19171-19184.

16 Szemraj M, Bielecka-Kowalska A, Oszajca K, Krajewska M, Gos R, Jurowski P, Kowalski M, Szemraj J: Serum MicroRNAs as Potential Biomarkers of AMD. Med Sci Monit 2015;21:2734-2742.

-17 Grassmann F, Schoenberger PG, Brandl C, Schick T, Hasler D, Meister G, Fleckenstein M, Lindner M, Helbig $\mathrm{H}$, Fauser S, Weber BH: A circulating microrna profile is associated with late-stage neovascular age-related macular degeneration. PloS one 2014;9:e107461.

18 Ertekin S, Yildirim O, Dinc E, Ayaz L, Fidanci SB, Tamer L: Evaluation of circulating miRNAs in wet agerelated macular degeneration. Mol Vis 2014;20:1057-1066.

19 Cao L, Wang H, Wang F, Xu D, Liu F, Liu C: Abeta-induced senescent retinal pigment epithelial cells create a proinflammatory microenvironment in AMD. Invest Ophthalmol Vis Sci 2013;54:3738-3750.

20 Lin H, Qian J, Castillo AC, Long B, Keyes KT, Chen G, Ye Y: Effect of miR-23 on oxidant-induced injury in human retinal pigment epithelial cells. Invest Ophthalmol Vis Sci 2011;52:6308-6314.

21 Zhou Q, Gallagher R, Ufret-Vincenty R, Li X, Olson EN, Wang S: Regulation of angiogenesis and choroidal neovascularization by members of microRNA-23 27 24 clusters. Proc Natl Acad Sci USA 2011;108:82878292. Wang L, Ye X, Liu Y, Wei W, Wang Z: Aberrant regulation of FBW7 in cancer. Oncotarget 2014;5:2000-2015. Chen Q, Xu J, Li L, Li H, Mao S, Zhang F, Zen K, Zhang CY, Zhang Q: MicroRNA-23a/b and microRNA-27a/b suppress Apaf-1 protein and alleviate hypoxia-induced neuronal apoptosis. Cell Death Dis 2014;5:e1132.

-24 Armstrong DA, Green BB, Seigne JD, Schned AR, Marsit CJ: MicroRNA molecular profiling from matched tumor and bio-fluids in bladder cancer. Mol Cancer 2015;14:194.

-25 Qiu F, Sun R, Deng N, Guo T, Cao Y, Yu Y, Wang X, Zou B, Zhang S, Jing T, Ling T, Xie J, Zhang Q: miR-29a/b enhances cell migration and invasion in nasopharyngeal carcinoma progression by regulating SPARC and COL3A1 gene expression. PLoS One 2015;10:e0120969.

-26 Zhang Y, Zhang D, Wang F, Xu D, Guo Y, Cui W: Serum miRNAs panel (miR-16-2*, miR-195, miR-2861, miR497) as novel non-invasive biomarkers for detection of cervical cancer. Sci Rep 2015;5:17942.

-27 Chen X, Shi K, Wang Y, Song M, Zhou W, Tu H, Lin Z: Clinical value of integrated-signature miRNAs in colorectal cancer: miRNA expression profiling analysis and experimental validation. Oncotarget 2015;6:37544-37556.

28 Chen HY, Zhong X, Huang XR, Meng XM, You Y, Chung AC, Lan HY: MicroRNA-29b inhibits diabetic nephropathy in db/db mice. Mol Ther 2014;22:842-853. 